\title{
Estudo sobre os fatores relacionados a interrupção do tratamento do glaucoma
}

\section{Study of the factors related to stop the treatment of glaucoma}

Eduardo Scaldini Buscacio', Giovanni Nicola Umberto Italiano Colombini²

\section{ReSUMO}

Objetivo: Identificar causas relacionadas com a não aderência ao tratamento do glaucoma primário de ângulo aberto e sugerir meios para posteriormente minimizá-las. Métodos: Foi aplicado um questionário a pacientes portadores de glaucoma primário de ângulo aberto no Hospital Universitário Gaffrée e Guinle, escolhidos aleatoriamente, para avaliação dos fatores relacionados com a interrupção do tratamento. Para isso, utilizou-se uma análise univariada, pelo teste exato de Fisher, e considerou estatisticamente significativo $p<0,05$. Resultados: A partir do questionário, identificou-se dois subgrupos, um que já havia interrompido o tratamento e outro que nunca o havia interrompido, compostos por 25 e 11 pacientes respectivamente. Estes grupos foram comparados entre si e todos os parâmetros analisados. $O$ custo dos medicamentos $(p=0,001)$ e o fator esquecimento $(p=0,007)$ foram estatisticamente relevantes para a interrupção do tratamento da doença. As demais variáveis testadas não obtiveram significância estatística. Conclusão: $\mathrm{O}$ custo dos medicamentos e o fator esquecimento foram os fatores mais importantes para interrupção do tratamento.

Descritores: Glaucoma/terapia; Adesão ao tratamento; Glaucoma primário de ângulo aberto/terapia; Custos de medicamentos; Suspensão de tratamento

\begin{abstract}
The objective was to identify causes related to noncompliance of primary open-angle glaucoma and suggest ways to minimize them later. A questionnaire was given to patients with primary open angle glaucoma in Hospital Gaffrée Guinle, chosen randomly, to assess factors related to discontinuation of treatment. For this we used a univariate analysis by Fisher's exact test and considered statistically significant $\mathrm{p}<0.05$. From the questionnaire, we identified two sub-groups, who had stopped treatment and another who had never stopped for 25 compounds and 11 patients respectively. These groups were compared, and all parameters examined. The cost of drugs $(\mathrm{p}=0.001)$ and forgetting factor $(p=0.007)$ were statistically significant for discontinuation of treatment of disease. The other variables tested did not achieve statistical significance. Conclusion: The cost of drugs and forgetting to take medication were the factors most important to withholding treatment treatment

Keywords: Glaucoma/therapy; Medication adherence; Glaucoma, open angle/therapy; Drug costs; Withholding

${ }^{1}$ Residente Médico do Hospital Municipal da Piedade - Rio de Janeiro (RJ), Brasil;

${ }^{2}$ Doutor; professor associado Universidade Federal do Estado do Rio de Janeiro - (UNIRIO) - Rio de Janeiro (RJ), Brasil.

Hospital Universitário Gaffrée e Guinle, Universidade Federal do Estado do Rio de Janeiro - (UNIRIO) - Rio de Janeiro (RJ), Brasil

Os autores declaram inexistir conflitos de interesse

Recebido para publicação em: 6/7/2010 - Aceito para publicação em 19/10/2011
\end{abstract}




\section{INTRODUÇÃO}

$\mathbf{G}$ laucoma é uma doença de causa desconhecida caracterizada pela degeneração dos axônios das células ganglionares da retina, podendo levar à cegueira irreversível ${ }^{(1)}$. É uma neuropatia óptica crônica progressiva, caracterizada por alterações típicas do disco óptico e da camada de fibras nervosas da retina, com repercussões características no campo visual. Na maioria das vezes está associado a valores de pressão intraocular (PIO) acima dos níveis considerados estatisticamente normais ${ }^{(1)}$. Existe uma minoria de casos de glaucoma, que evoluem com PIO normal. Estima-se uma prevalência de $0,2 \%$ da população na quinta década de vida e 1,6\% em torno dos 75 anos de idade ${ }^{(2)}$. Apesar da relação causa/efeito não ser muito precisa, sabe-se que o aumento da PIO é o principal fator de risco para desenvolver a neuropatia óptica glaucomatosa $^{(1-4)}$.

O tratamento do glaucoma visa a prevenir o déficit visual definitivo e, dessa forma, a aderência dos pacientes ao tratamento tem importantes implicações sociais ${ }^{(5)}$. Estudos anteriores mostraram que uma parcela significativa dos pacientes interrompem a terapia. Nordstrom et al ${ }^{(5)}$ reportam relação entre a interrupção do tratamento e o tipo de droga utilizada, enquanto Costa et al.$^{\left({ }^{()}\right.}$e Silva et al.$^{(7)}$ associam a não adesão ao baixo conhecimento a respeito da doença e ao custo dos medicamentos.

Este estudo teve como objetivo avaliar os principais fatores relacionados à não aderência dos pacientes ao tratamento do GPAA, visando acrescentar ao conhecimento médico, medidas que possam evitar tais fatores.

\section{Métodos}

No Hospital Universitário Gaffrée Guinle, da Universidade Federal do Estado do Rio de Janeiro, Brasil, foi realizado um estudo de caso controle aplicando em portadores de GPAA um questionário para avaliação dos principais fatores relacionados com a interrupção do tratamento desta afecção.

O questionário consta de cabeçalho com preenchimento de dados pessoais como idade, sexo, raça e ocupação, três perguntas objetivas visando avaliar o conhecimento do paciente sobre a doença, duas questões objetivas e três subjetivas procurando estabelecer aproximadamente as condições financeiras do entrevistado, duas questões objetivas e quatro subjetivas sobre as dro- gas já utilizadas pelo paciente desde o primeiro diagnóstico de glaucoma, duas questões objetivas sobre a aderência do paciente propriamente dita e uma pergunta sobre a relação do entrevistado com o médico oftalmologista (Apêndice 1).

Foram adotados os seguintes critérios para inclusão de pacientes: diagnóstico de glaucoma confirmado; acompanhamento ambulatorial na instituição por um período mínimo de 1 ano; maiores de 40 anos; podendo ter realizado ou não cirurgia antiglaucomatosa; concordância na participação do estudo por livre consentimento.

Foram adotados os seguintes critérios para exclusão de pacientes: a não concordância com a participação do estudo; diagnóstico de glaucoma congênito, secundário e de ângulo fechado.

A partir do questionário, os pacientes foram divididos em 2 subgrupos: um subgrupo contendo pacientes que já interromperam em algum momento o tratamento, tendo voltado ou não a tratar, e outro subgrupo com os que nunca interromperam. Estes 2 subgrupos foram comparados procurando-se variáveis que justificassem a não adesão ao tratamento. Considerou-se diferenças significativas entre os 2 subgrupos quando na análise univariada encontrou-se $\mathrm{p}<0,05$, através do Teste exato de Fisher, numa comparação de variáveis duas a duas.

\section{Resultados}

A amostra foi composta por 36 pacientes com idades variando entre 41 e 86 anos, com média de 66,63 anos e com desvio padrão de 11,87 anos. Dentre esses, 26 pacientes eram do sexo feminino representando $72,2 \%$ dos entrevistados, e 10 pacientes eram do sexo masculino representado $27,8 \%$ da amostra. Quanto à atividade laborativa dos pacientes, 20 (55\%) eram aposentados, 8 $(22 \%)$ eram do lar, e cada entrevistado restante $(2,7 \%)$ se ocupava com as seguintes profissões, assistente administrativa, auxiliar de máquina, comerciante, cozinheira, lavadeira, profissional liberal, operador de máquinas e vigilante.

Quanto à adesão ao tratamento, observou-se que 11 pessoas $(30,6 \%)$ sempre usaram os colírios corretamente e que 25 pessoas $(69,4 \%)$ já haviam deixado de usar o colírio antiglaucomatoso em algum momento desde que foram diagnosticados. Foram analisados fatores como o custo dos medicamentos, o fator esquecimento, dificuldades não financeiras em adquirir o medicamento e os efeitos colaterais com os resultados descritos na tabela 1 . 
Tabela 1

Fatores de não adesão ao tratamento

\begin{tabular}{lccc}
\hline Fatores & $\begin{array}{c}\text { Pacientes } \\
\text { que aderiram ao } \\
\text { tratamento (\%) }\end{array}$ & $\begin{array}{c}\text { Pacientes } \\
\text { que interromperam } \\
\text { o tratamento (\%) }\end{array}$ & $\begin{array}{c}\text { Análise } \\
\text { univariada } \\
\text { Valor de p }\end{array}$ \\
\hline $\begin{array}{l}\text { Custo dos medicamentos } \\
\text { Sim }\end{array}$ & 0 & $15(60)$ & \\
Não & $11(100)$ & $10(40)$ & 0,001 \\
$\begin{array}{l}\text { Esquecimento } \\
\text { Sim }\end{array}$ & 0 & $8(32)$ & \\
Não & $11(100)$ & $17(68)$ & 0,007 \\
Dificuldade em adquirir a medicação & 0 & $3(12)$ & \\
Sim & $11(100)$ & $22(88)$ & 0,538 \\
Não & 0 & $2(8)$ & \\
Efeitos colaterais & $11(100)$ & $23(92)$ & 1,000 \\
Sim & & & \\
Não & & &
\end{tabular}

Tabela 2

Dados epidemiológicos

\begin{tabular}{lccc}
\hline Fatores & $\begin{array}{c}\text { Pacientes } \\
\text { que aderiram } \\
\text { ao tratamento (\%) }\end{array}$ & $\begin{array}{c}\text { Pacientes } \\
\text { que interromperam } \\
\text { o tratamento (\%) }\end{array}$ & $\begin{array}{c}\text { Análise } \\
\text { Univariada } \\
\text { (valor de p) }\end{array}$ \\
\hline $\begin{array}{l}\text { Instrução fundamental } \\
\text { Completa }\end{array}$ & $3(27)$ & $5(20)$ & \\
$\begin{array}{l}\text { Incompleta } \\
\text { Sexo }\end{array}$ & $8(73)$ & $20(80)$ & 0,678 \\
$\begin{array}{l}\text { Masculino } \\
\text { Feminino }\end{array}$ & $4(36)$ & $6(24)$ & \\
$\begin{array}{l}\text { Renda familiar } \\
>1000 \text { reais } \\
<1000 \text { reais }\end{array}$ & $7(64)$ & $19(76)$ & 0,454 \\
& $6(55)$ & $13(52)$ & \\
& $5(45)$ & $12(48)$ & 1,000 \\
\hline
\end{tabular}

Dados epidemiológicos como o grau de escolaridade, o sexo, o nível socioeconômico, definido de acordo com a renda familiar(maior que mil e menor que mil reais) também foram avaliados e os resultados podem ser observados na tabela 2.

Na tabela 3 observam-se os resultados que arguiram quanto ao conhecimento sobre a patologia em estudo. Variáveis como "autoavaliação", na qual o próprio paciente graduava seu nível de conhecimento sobre a doença entre Pouco/Nenhum e Médio/Profundo, como também através de perguntas objetivas sobre o glaucoma, como a lesão do nervo óptico, correlação com a PIO e a eventual possibilidade de cura, também foram testadas.

Na tabela 4 demonstra-se os resultados a respeito das drogas utilizadas no tratamento do glaucoma. Fo- ram testadas separadamente as seguintes classes de drogas: alfa agonistas, inibidores da anidrase carbônica, prostamidas e betabloqueadores. Também foram variáveis a frequência do uso e a manutenção ou não da mesma medicação.

Quando questionados sobre a relação com o oftalmologista, dentre os pacientes com má adesão 14 $(56,0 \%)$ afirmaram uma ótima relação, $9(36,0 \%)$ como boa e $2(8,0 \%)$ classificaram como média. Já no outro grupo, $7(63,6 \%)$ classificaram como ótima e $4(36,4 \%)$ como boa.

De todos os parâmetros analisados, o custo dos medicamentos e fator esquecimento foram estatisticamente relevantes para a interrupção do tratamento da doença, utilizando-se uma análise univariada, pelo teste de Fisher $(\mathrm{p}<0,05)$ com nível de significância 95\%. 
Tabela 3

Conhecimento sobre a doença

\begin{tabular}{lccc}
\hline Fatores & $\begin{array}{c}\text { Pacientes } \\
\text { que aderiram } \\
\text { ao tratamento (\%) }\end{array}$ & $\begin{array}{c}\text { Pacientes } \\
\text { que interromperam } \\
\text { o tratamento (\%) }\end{array}$ & $\begin{array}{c}\text { Análise } \\
\text { univariada } \\
\text { Valor de p }\end{array}$ \\
\hline $\begin{array}{l}\text { Auto avaliação } \\
\text { Profundo/Médio }\end{array}$ & $5(55)$ & $8(32)$ & \\
$\begin{array}{l}\text { Pouco/Nenhum } \\
\text { Leva a cegueira }\end{array}$ & $6(45)$ & $17(68)$ & 0,475 \\
$\begin{array}{l}\text { Sim } \\
\text { Não }\end{array}$ & $9(81)$ & $23(92)$ & \\
Relação com aumento da PIO & $2(19)$ & $2(8)$ & 0,570 \\
Sim & $9(81)$ & $25(100)$ & \\
Não & $2(19)$ & $0(0)$ & 0,087 \\
Lesão do nervo óptico & $3(27)$ & $12(48)$ & \\
Sim & $8(73)$ & $13(52)$ & 0,295 \\
Não & & $4(16)$ & \\
Doença tem cura & $4(36)$ & $21(84)$ & 0,214 \\
Sim & $7(64)$ & & \\
Não & &
\end{tabular}

Tabela 4

Drogas utilizadas no tratamento

\begin{tabular}{|c|c|c|c|}
\hline Fatores & $\begin{array}{c}\text { Pacientes } \\
\text { que aderiram } \\
\text { ao tratamento }(\%)\end{array}$ & $\begin{array}{c}\text { Pacientes } \\
\text { que interromperam } \\
\text { o tratamento }(\%)\end{array}$ & $\begin{array}{c}\text { Análise } \\
\text { univariada } \\
\text { Valor de p }\end{array}$ \\
\hline \multicolumn{4}{|c|}{ Troca da medicação } \\
\hline Sim & $7(64)$ & $15(60)$ & \\
\hline Não & $4(36)$ & $10(40)$ & 1,000 \\
\hline \multicolumn{4}{|c|}{ Frequência de uso } \\
\hline$<3 \mathrm{x} /$ dia & $5(45)$ & $15(60)$ & 0,483 \\
\hline \multicolumn{4}{|c|}{ Uso de alfa agonistas } \\
\hline Sim & $2(18)$ & $6(24)$ & \\
\hline Não / Não sabe & $9(82)$ & $19(76)$ & 1,000 \\
\hline \multicolumn{4}{|c|}{ Uso de inibidor da anidrase carbônica } \\
\hline Sim & $4(36)$ & $6(24)$ & \\
\hline Não / Não sabe & $7(64)$ & $19(76)$ & 0,454 \\
\hline \multicolumn{4}{|c|}{ Uso de prostamidas } \\
\hline Sim & $6(55)$ & $11(44)$ & \\
\hline Não / Não sabe & $5(45)$ & $14(56)$ & 0,721 \\
\hline \multicolumn{4}{|c|}{ Uso de beta antagonistas } \\
\hline Sim & $7(64)$ & $15(60)$ & \\
\hline Não / Não sabe & $4(36)$ & $10(40)$ & 1,000 \\
\hline
\end{tabular}




\section{APÊNDICE 1}

QUESTIONÁRIO BASE

\section{Questionário:}

(1) Dados pessoais:

Nome completo:

Idade em anos completos:

Sexo: ( ) masculino ( ) feminino

Profissão:

Endereço (Rua, Av.)

№

Compl

Bairro

Município

Estado

Naturalidade

Nacionalidade

Grau de escolaridade:

O Sr (a) já estudou? ( ) Sim ( ) Não

Se sim: até que série o sr.(a) completou? Escolha uma das opções abaixo:

A: Nunca estudou

B: Sabe apenas assinar o nome

C: pré-escolar

D: Fundamental incompleto
D1: 1a série
D2: $2^{\mathrm{a}}$ série
D3: $3^{\text {a }}$ série
D4: 4⿳亠丷厂 série
D5: 5a série
D6: 6aㅗ série
D7: $7^{\text {a }}$ série
D8: ${ }^{\text {a }}$ série

E: Fundamental completo

F: Médio incompleto

F1: $1^{\circ}$ ano

F2: $2^{\circ}$ ano

F3: $3^{\circ}$ ano

G: Médio completo

$\mathrm{H}$ : Superior incompleto

I: Superior completo

2) Qual o seu grau de conhecimento a respeito do glaucoma?

( ) Nenhum ( ) Pouco ( ) Médio ( ) Profundo conhecedor

3)Quais das alterações abaixo podem acontecer no glaucoma?

( ) Coceira

( ) Olho saltado

( ) Olho esbranquiçado

( ) Doença no nervo óptico

( ) Cegueira

( ) Diminuição da visão

( ) Pressão alta no olho

( ) Dor no olho

( ) Lesão do disco óptico

( ) Remela 
4) Qual a importância de realizar o tratamento adequado?

( ) Melhorar a visão

( ) Evitar progressão da doença

( ) Curar

( ) Evitar ficar cego

( ) Evitar outras doenças

( ) Não tem muita importância

5) Atualmente o sr.(a) trabalha e recebe algum pagamento pelo seu trabalho?

( ) $\operatorname{Sim}($ ) Não

6) Se recebe, quantos reais o sr.(a) ganha por mês?

7) Na sua casa, além do sr.(a) alguém mais trabalha e ajuda nas despesas do mês?

( ) Sim ( ) Não

8) Quantas pessoas? ( )

9) Quanto é em média a sua renda familiar? ( )

10) Qual(is) o(s) colírio(s) que o sr.(a) usa atualmente para o tratamento do glaucoma?

11) Com que frequência o sr.(a) pinga o(s) colírio (s)?
( ) $1 x$ ao dia
( ) $2 x$ ao dia
( ) $3 x$ ao dia
( ) Quando lembra
( ) $1 \times$ por semana
( ) $2 x$ por semana
( ) Outro
12) Em que horário(s) o sr.(a) costuma pingar o(s) colírio(s)?

13) O sr.(a) tem ou já teve algum tipo de efeito colateral com o uso do colírio? S/N

14) O sr.(a) já usou outro tipo de colírio para o tratamento do glaucoma? S/N

15) Caso já tenha usado, porque trocou a medicação?

( ) Efeitos colaterais

( ) Muito caro

( ) Falta de melhora no tratamento

( ) outros

16) O sr.(a ) desde que começou o tratamento já deixou em algum momento de usar a medicação? $\mathrm{S} / \mathrm{N}$

17) Caso já tenha deixado, porque interrompeu o uso da medicação?

( ) Efeitos colaterais

( ) Muito caro

( ) Dificuldade de adquirir a medicação

( ) Falta de melhora no tratamento

( ) Outros

18) Como o sr.(a) classificaria a sua relação com o seu médico oftalmologista?
( ) Ótima
( ) Boa
( ) Média
( ) Ruim
( ) Péssima 


\section{DıscussÃo}

No presente estudo, investigaram-se as variáveis relacionadas à má adesão dos pacientes ao tratamento do GPAA. O tamanho da amostra ficou limitado ao volume de pacientes atendidos no setor, podendo o tamanho da amostra ter influenciado na significância dos resultados. Mais da metade dos entrevistados $(69,4 \%)$ assumiu ter alguma falha na adesão desde que descobriram ter a doença, sendo que maioria atribuiu a má adesão ao fato de o colírio ter um alto custo. Este parâmetro foi estatisticamente significante, conforme pode-se observar na tabela 1 , estando compatível com pesquisas anteriores ${ }^{(6,7)}$.

Sabe-se que existem diversos fatores sociais que podem justificar esse resultado, porém, a falta de distribuição dos colírios nas farmácias públicas somado a um baixo poder aquisitivo da população, podem ser fatores importantes na gênese da descontinuidade da terapia ${ }^{(7)}$. Neste estudo, observou-se também uma renda individual média e a renda familiar média um pouco maior no grupo que sempre aderiu quando comparado ao grupo que tinha falha na adesão. Este resultado não apresentou significância estatística, diferindo de trabalhos anteriores ${ }^{(6,7)}$.

Entre os outros pontos também pesquisados destaca-se o fator esquecimento, o qual obteve da significância estatística, sendo causa presente em 32\% dos pacientes que não aderiram ao tratamento. Estando de acordo com estudos ${ }^{(8)}$. Tal fato sugere que o médico oftalmologista deve sempre informar o paciente sobre o seu tratamento e a importância de fazê-lo corretamente. Outras variáveis analisadas que se associaram à não adesão ao tratamento como a dificuldade em adquirir a medicação, presentes como causa em $12 \%$, e os efeitos colaterais, presentes em $8 \%$, também já foram descritas anteriormente ${ }^{(8)}$.

Ao se avaliar as variáveis relacionadas ao nível instrução dos entrevistados, observou-se que o nível de escolaridade no grupo que tinha descontinuidade no tratamento, era inferior do que no grupo que sempre aderiu, já que o primeiro tinha uma porcentagem maior de pacientes que nunca estudaram e que não tinham completado o ensino fundamental. Em relação ao conhecimento do paciente sobre o glaucoma, notou-se que o grupo que sempre aderiu, tinha um conhecimento ligeiramente menor sobre a doença que o outro grupo, visto que uma menor porcentagem de indivíduos deste grupo sabia do acometimento do nervo e do disco óptico, e uma porcentagem maior deste grupo acreditava ser possível curar e melhorar a acuidade visual com o tratamento. Não foi obtido significância estatística nesses critérios no presente estudo. Estes resultados, por sua vez, divergem de outros publicados anteriormente ${ }^{(9)}$.

Ao se avaliar as variáveis relacionadas à droga, a grande maioria dos pacientes dos dois grupos já havia usado outras medicações, sendo o motivo de troca da medicação atribuído principalmente a falha terapêutica . Nenhum fármaco foi associado à não adesão terapêutica. Este resultado não obteve significância estatística, divergindo de resultados anteriores nos quais os pacientes que faziam uso análogos da prostaglandina tinham uma maior adesão ao tratamento ${ }^{(5)}$, não observou-se diferença quanto as drogas.

\section{CONCLUSÃo}

O presente estudo identificou padrões associados a interrupção do tratamento do GPAA, dentre os quais, os únicos com significância, foram o custo dos medicamentos e o fator esquecimento.

\section{REFERÊNCIAS}

1. Sociedade Brasileira de Glaucoma. $2^{\circ}$ Consenso Brasileiro de Glaucoma Primário de Ângulo Aberto. São Paulo: PlanMark; 2005. p. 9.

2. Tavares IM, Mello PAA. Glaucoma de pressão normal. Arq Bras Oftalmol. 2005;68(4):565-75.

3. European Glaucoma Society. Terminology and guidelines for glaucoma. 3rd ed. Savona: Dogma; 2008.

4. Allingham RR, Damji KF, Freedman S, Moroi SE, Shafranov G. Shields' textbook of glaucoma. 5th ed. Philadelphia: Lippincott Williams \& Wilkins; 2005.

5. Nordstrom BL, Friedman DS, Mozaffari E, Quigley HA, Walker AM. Persistence and adherence with topical glaucoma therapy. Am J Ophthalmol. 2005;140(4):598-606.

6. Costa VP, Vasconcellos JPC, Pelegrino M, José NK. O que os pacientes sabem sobre glaucoma? Arq Bras Oftalmol. 1995;58(1):36-41.

7. Silva LMS, Vasconcellos JPC, Temporini ER, Costa VP, KaraJosé N. Tratamento clínico do glaucoma em um hospital universitário: custo mensal e impacto na renda familiar. Arq Bras Oftalmol. 2002;65(3):299-303.

8. Yasuoka ER, Mello PAA, Norris EJ. Quem segue corretamente o tratamento clínico do glaucoma? Arq Bras Oftalmol. 1996;59(2):199-204.

9. Silva MJL, Temporini ER, Neustein I, Araújo MEXS. Conhecimentos sobre prevenção e tratamento de glaucoma entre pacientes de unidade hospitalar. Arq Bras Oftalmol. 2004;67(5):785-90.

\footnotetext{
Endereço para correspondência:

Eduardo Scaldini Buscacio

Rua Senador Vergueiro, $n^{\circ} 93$ - apto 503 - Flamengo

CEP 22230-000 - Rio de Janeiro (RJ), Brasil.

E-mail: eduardosbuscacio@gmail.com
} 